\title{
Asset Allocation, Time Diversification and Portfolio Optimization for Retirement
}

\author{
Kamphol Panyagometh \\ NIDA Business School, Bangkok, Thailand \\ E-mail: kpanyagometh@gmail.com, kpanyago@hotmail.com \\ Received February 17, 2011; revised April 6, 2011; accepted April 10, 2011
}

\begin{abstract}
Using the data of stock, commodity and bond indexes from 2002 to November 2010, this research was carried out by employing Bootstrapping Simulation technique to find an optimal portfolio (portfolio optimization) for retirement, and the effect of diversification based on increased length of investment period (time diversification) with respect to the lengths of retirement investment period and the amounts required for spending after retirement in various occasions. The study analyzed for an optimal allocation of common stock, commodity and government bond to achieve the target rate of return for retirement by minimizing the portfolio risk as measured from the standard deviation. Apart from the standard deviation of the rate of return of the investment portfolio, this study also viewed the risk based on the Value at Risk concept to study the downside risk of the investment portfolio for retirement.
\end{abstract}

Keywords: Asset Allocation, Time Diversification, Portfolio Optimization, Bootstrapping Simulation

\section{Introduction}

Nowadays the structure of Thai society is one with a growing proportion of the elderly. While medical technology has experienced a rapid pace of advancement, the average age of the population is increasing; which means that Thai people need to be planning to save money for longer retirement life. Data available at the National Statistical Office (NSO) showed that 73 percent of the overall population was not a member of any retirement fund; where only 23.3 percent was. Therefore, the government devised a policy to push for saving fund establishment in the form of retirement saving fund on the grounds that, in the long run, the elderly with neither money nor security shall become a burden to family members while governmental welfare might not be thoroughly accessible. For this reason, the Thai society should place importance on the systematic, conventional, and consistent long term investment and saving system especially during its working years, where it should start to save and invest through funds or investment and saving systems under expert supervision in order to be able to manage its investment for generating income stream during retirement efficiently.

This research was carried out to find an optimal investment ratio between common stock, commodity and bond in investment portfolio (portfolio optimization) for retirement, and the effect of diversification based on increased length of investment period (time diversification). The study employed Bootstrapping Simulation technique to generate long term rates of return with respect to the lengths of retirement investment period and the amounts required for spending after retirement in various occasions; and analyzed for an optimal investment ratio between common stock and government bond to achieve the target rate of return for retirement by minimizing the portfolio risk as measured from the standard deviation. Apart from the standard deviation of the rate of return of the investment portfolio, this study also viewed the risk based on the Value at Risk concept to study the downside risk of the investment portfolio for retirement.

The rest of the paper is organized as follows. Section 2 reviews related literature. Section 3 discusses the research data used. Section 4 explains research methodology and Bootstrapping simulation technique. Section 5 discusses the results of the study, and Section 6 concludes.

\section{Literature Review}

Levy [1] illustrated the time diversification phenomenon by studying the past performance of common stocks and 
government bonds at different lengths of investment period, and found that common stocks offered better rate of return than government bonds did in all investments with 25 years period. This outcome held true during the investment years 1926-1977. Reichenstein [2] employed the same techniques and research methodologies as Levy [1] did and proved that the risk of investment portfolio was dependent on the investment period. Leibowitz and Langetieg [3] developed a model under the hypothesis that common stocks had a risk premium of $4 \%$ higher than that of debentures. This risk premium value was less than the actual value obtained from the data since 1926, where common stocks had a risk premium of $7 \%$ higher than that of debentures. This study showed that while the risk premium became less, the benefits of common stocks as compared with debentures became lower for both short-term and long-term investments. Butler and Domian [4] studied a long-term rate of return of retirement portfolios of common stocks and debentures by attempting various asset allocation strategies. The outcome revealed that as the length of pre-retirement investment period was increased, common stock investment risk was greatly reduced as compared with that of debentures; and when the pre-retirement investment period reached or exceeded 30 years, the chance that common stocks would yield less than debentures would be less than 4\%. Bjorn and Persson [5] suggested that long-term investors should invest in a high proportion of risky assets. The study showed that efficient portfolios with long investment period would have a higher proportion of share investment as compared with efficient portfolios with only 1 year investment period. Strong and Taylor [6] examined the relationship between security returns and investment periods, and pointed out the time diversification phenomenon of optimal investment portfolio. Hickman, Hunter, Byrd, Beck, and Terpening [7] employed Bootstrapping technique in the monthly rate of return study during 1926-1997 and found that the chance for monthly investment in common stocks to yield less than government bonds dropped from $39 \%$ to $6 \%$ when the pre-retirement investment period increased from 1 year to 30 years. Gollier [8] proposed a theory of time diversification and absolute risk aversion for wealth. The research found that time diversification phenomenon would occur in the case where investors discriminated between wealth-related risk and consumption-related risk. Howe and Mistic [9] studied the impact of personal income tax on the return of various asset classes on the retirement date. Guo and Darnell [10] tried to prove the existence of time diversification by using past returns data of S\&P500, T-bill, and T-Bond indices during 1802-2002. This study found that the benefits of time diversification depended on the risk of security index return rate when compared with the risk of government bond return rate. Mukherji [11] published the study outcome which contributed to time diversification research efforts through the use of Block Bootstrap method to model long-term rate of returns and analyze the investment diversification of 6 categories of financial assets to find an optimal investment portfolio by minimizing the downside risk based on a number of required rates of return. Alles [12] studied the effect of downside protection development cost and time diversification among Stock Exchange investors in Bangladesh, Sri Lanka, India, and Pakistan using Bootstrapping methodology where the investment period ranged from 1 to 20 years. It was found that the cost of downside protection varied from country to country, and was lessened with the increase in the length of investment period. This is consistent with the benefit of time diversification. Panyagometh [13] demonstrated the benefit of time diversification by showing that an investor's starting the retirement investment early in order to have a longer pre-retirement investment period not only reduced the monthly pre-retirement investment amount, but also mitigated the probability of ruin associated with the retirement investment.

\section{Research Data}

This study employed the 2002-November 2010 data comprising the Stock Exchange of Thailand total return index (SET TRI) calculated by the Stock Exchange of Thailand, representing investment in common stock asset class; the Rogers International Commodity Index (RICI $\left.{ }^{\circledR}\right)$ calculated by Beeland Interests, Inc., representing investment in commodity asset class; and the government bond total return index (TBMA total return index, BOND TRI) calculated by the Thai Bond Market Association, representing investment in government bond asset class.

SET TRI is an index calculated from all types of return on security investment so as to have all of them reflected; namely, returns based on capital gain/loss; rights to subscribe for shares, which is granted for existing shareholders to buy new shares usually at the price lower than their market price at that moment; and dividends, which are the share of profits paid to shareholders, with supplemental assumption that the dividends received shall again be invested in securities (reinvest).

RICI ${ }^{\circledR}$ is a composite, USD based, total return index calculated from 37 commodities from 13 international exchanges. It represents the value of a basket of commodities consumed in the global economy, ranging from agricultural to energy and metals products. The index is divided into three sub-indices, which reflect the three sub-segments of the RICI - RICI Agriculture, RICI En- 
ergy and RICI Metals. The sub-indices' contribution to main index from the beginning are Agriculture - 34.90\%, Energy $-44.00 \%$, Metals $-21.10 \%$ according to the RICI Handbook.

BOND TRI is an index obtained through the same calculation methodology as the index of European of Financial Analysts Societies, or EFFAS; which is based on international principle. The calculation makes use of all government bonds which are registered bonds listed at the Thai Bond Dealing Centre; not including state owned enterprise bonds, Bank of Thailand bonds, Financial Institution Development Fund bonds, etc. in order for the index to exclusively reflect the market situation of government bonds, which are deemed truly risk-free with regard to default in payment of interest and principal. The weighted average executed yield is used in the calculation, where each item of the executed yield is weighted by its value traded. After the weighted average executed yield is obtained, the clean price of each bond is then calculated. The resulting index shall therefore represent the movements of government bond market on a daily basis, where BOND TRI includes the interest due on the index calculation date within its calculation.

Table 1 presents SET TRI, RICI® and BOND TRI monthly data during 2002 - November 2010. Table 2 shows statistics information of the research data presenting the average monthly (annual) return on investment in SET TRI, RICI ${ }^{\circledR}$ and BOND TRI of $1.57 \%(18.84 \%)$, $0.75 \%(9.00 \%)$ and $0.41 \%(4.92 \%)$ respectively; where the standard deviation of the monthly (annual) return on investment in SET TRI, RICI ${ }^{\circledR}$ and BOND TRI reads $6.76 \%(23.42 \%), 5.46 \%$ (18.91\%) and $1.90 \%(6.58 \%)$. As foreseen, common stock is an asset which provides a higher return than bond, but it has a higher risk as well. With regard to the value -0.1160 of the correlation coefficient between common stock and bond, the value -0.2820 of the correlation coefficient between bond and commodity, and the value 0.3771 of the correlation coefficient between stock and commodity, investors who invest in common stock, commodity and bond in their investment portfolio shall be able to benefit from diversification since these asset classes have low or even negative correlation coefficients.

\section{Research Methodology: Bootstrapping Simulation}

Bootstrapping Simulation technique involves randomly and repeatedly sampling values of data in order to estimate the distributions of required statistics. Bootstrapping Simulation can reduce the risk of assessment error occurrence when the true parameters of the rate of return distribution are unknown. Singh [14] showed that the distribution of data estimated by Non-parametric Bootstrap method has high degree of accuracy.

Steps to Performing Bootstrapping Simulation:

Suppose we want to run Bootstrapping Simulation to model a return on monthly investment of 1 Baht per month in SET TRI, RICI ${ }^{\circledR}$, and BOND TRI for a period of 5 years or 60 months:

Step 1: Assign codes to the monthly rate of return data of SET TRI, RICI ${ }^{\circledR}$ and BOND TRI during 2002 - November 2010 as shown in Table 1.

Step 2: Simulate the monthly return of SET TRI, RICI ${ }^{\circledR}$ and BOND TRI in each month for 60 months. The monthly return of each month is obtained through generating a random number between 1 and 106, and using the return rate of SET TRI, RICI ${ }^{\circledR}$ and BOND TRI in the month which matches the number generated as monthly return for that month. An example of a simulation run is shown in Table $\mathbf{3}$ in which a random number generated for month 1 is 33, which corresponds to a monthly rate of return of $29 / 10 / 2004$, the value of which was $-2.54 \%, 0.78 \%$ and $-0.29 \%$ for SET TRI, RICI® and BOND TRI respectively; while a random number generated for month 20 is 3 , which corresponds to a monthly rate of return of $30 / 4 / 2002$, the value of which was $0.60 \%,-0.72 \%$ and $1.18 \%$ for SET TRI, RICI® and BOND TRI respectively. Through this method, the monthly rate of return of 60 months can be obtained, as shown in Table 3.

Step 3: Calculate the return amount from the investment in SET TRI, RICI ${ }^{\circledR}$ and BOND TRI for each month as shown in Table 3, starting from investing 1 Baht in month 0 (beginning of month 1 ). In month 1 , SET TRI offers $-2.54 \%$ rate of return, and RICI ${ }^{\circledR}$ offers $0.78 \%$, while BOND TRI offers $-0.29 \%$; thus after a month has passed the money invested in SET TRI is equal to $1 \times(1-$ $0.0254)=0.9746$, and the money invested in RICI ${ }^{\circledR}$ is equal to $1 \times(1+0.0075)=1.0075$ while the money invested in BOND TRI is equal to $1 \times(1-0.0029)=$ 0.9971. Then add 1 Baht monthly amount being invested each month yielding the result at the end of month 1 (beginning of month 2) of 1.9746, 2.0075 and 1.9971 for SET TRI, RICI ${ }^{\circledR}$ and BOND TRI respectively. In month 2 , SET TRI rate of return is $-0.96 \%$, and that of RICI ${ }^{\circledR}$ is $4.11 \%$ while that of BOND TRI is $-0.06 \%$; therefore, after another month has passed the investment in SET TRI is equal to $1.9746 \times(1-0.0096)=1.9556$, and the investment in RICI ${ }^{\circledR}$ is equal to $2.0075 \times(1+0.0411)=$ 2.0900 the while the investment in BOND TRI is equal to $1.9971 \times(1-0.0006)=1.9959$. Again add 1 Baht monthly amount being invested each month yielding the result at the end of month 2 (beginning of month 3 ) of 2.9556, 3.0900 and 2.9959 for SET TRI, RICI ${ }^{\circledR}$ and BOND TRI respectively. The calculation proceeds ac- 
Table 1. Monthly data of SET Total Return Index, Rogers International Commodity Index and ThaiBMA Government Bond Total Return Index during the year 2002-Nov 2010.

\begin{tabular}{|c|c|c|c|c|c|c|c|c|c|}
\hline \multirow{2}{*}{ Code } & \multirow{2}{*}{ Date } & \multicolumn{3}{|c|}{ MONTHLY RETURN } & \multirow{2}{*}{ Code } & \multirow{2}{*}{ Date } & \multicolumn{3}{|c|}{ MONTHLY RETURN } \\
\hline & & SET TRI & RICI $^{\circledR}$ & BOND TRI & & & SET TRI & RICI $^{\circledR}$ & BOND TRI \\
\hline 1 & $28 / 2 / 2002$ & $9.33 \%$ & $3.47 \%$ & $-0.69 \%$ & 54 & $31 / 7 / 2006$ & $1.98 \%$ & $0.70 \%$ & $1.18 \%$ \\
\hline 2 & $29 / 3 / 2002$ & $1.21 \%$ & $10.52 \%$ & $-2.07 \%$ & 55 & $31 / 8 / 2006$ & $0.78 \%$ & $-4.68 \%$ & $1.47 \%$ \\
\hline 3 & $30 / 4 / 2002$ & $0.60 \%$ & $-0.72 \%$ & $1.18 \%$ & 56 & 29/9/2006 & $-0.11 \%$ & $-6.47 \%$ & $1.35 \%$ \\
\hline 4 & $31 / 5 / 2002$ & $9.95 \%$ & $-2.24 \%$ & $0.53 \%$ & 57 & $31 / 10 / 2006$ & $5.38 \%$ & $-1.19 \%$ & $0.40 \%$ \\
\hline 5 & $28 / 6 / 2002$ & $-4.58 \%$ & $1.93 \%$ & $1.08 \%$ & 58 & $30 / 11 / 2006$ & $2.37 \%$ & $3.13 \%$ & $2.17 \%$ \\
\hline 6 & $31 / 7 / 2002$ & $-3.36 \%$ & $1.77 \%$ & $1.71 \%$ & 59 & $29 / 12 / 2006$ & $-7.96 \%$ & $-4.81 \%$ & $-2.41 \%$ \\
\hline 7 & $30 / 8 / 2002$ & $-3.75 \%$ & $5.53 \%$ & $2.79 \%$ & 60 & $31 / 1 / 2007$ & $-3.70 \%$ & $-1.99 \%$ & $3.53 \%$ \\
\hline 8 & $30 / 9 / 2002$ & $-8.02 \%$ & $5.49 \%$ & $1.87 \%$ & 61 & $28 / 2 / 2007$ & $3.61 \%$ & $3.23 \%$ & $1.99 \%$ \\
\hline 9 & $31 / 10 / 2002$ & $7.74 \%$ & $-1.93 \%$ & $0.84 \%$ & 62 & $30 / 3 / 2007$ & $0.48 \%$ & $0.34 \%$ & $1.47 \%$ \\
\hline 10 & $29 / 11 / 2002$ & $2.23 \%$ & $0.90 \%$ & $1.45 \%$ & 63 & $30 / 4 / 2007$ & $5.21 \%$ & $-0.14 \%$ & $2.50 \%$ \\
\hline 11 & $31 / 12 / 2002$ & $-2.19 \%$ & $4.71 \%$ & $0.60 \%$ & 64 & $31 / 5 / 2007$ & $5.70 \%$ & $0.17 \%$ & $0.35 \%$ \\
\hline 12 & $31 / 1 / 2003$ & $3.82 \%$ & $6.35 \%$ & $2.23 \%$ & 65 & $29 / 6 / 2007$ & $5.35 \%$ & $2.68 \%$ & $-2.52 \%$ \\
\hline 13 & $28 / 2 / 2003$ & $-2.02 \%$ & $5.88 \%$ & $0.01 \%$ & 66 & $31 / 7 / 2007$ & $10.68 \%$ & $2.55 \%$ & $0.06 \%$ \\
\hline 14 & $31 / 3 / 2003$ & $1.99 \%$ & $-6.81 \%$ & $-0.34 \%$ & 67 & $31 / 8 / 2007$ & $-4.79 \%$ & $-1.29 \%$ & $-0.19 \%$ \\
\hline 15 & $30 / 4 / 2003$ & $4.62 \%$ & $-3.94 \%$ & $0.35 \%$ & 68 & $28 / 9 / 2007$ & $4.46 \%$ & $9.25 \%$ & $0.50 \%$ \\
\hline 16 & $30 / 5 / 2003$ & $7.93 \%$ & $5.48 \%$ & $2.00 \%$ & 69 & $31 / 10 / 2007$ & $7.35 \%$ & $5.42 \%$ & $-0.41 \%$ \\
\hline 17 & $30 / 6 / 2003$ & $14.40 \%$ & $1.74 \%$ & $2.25 \%$ & 70 & $30 / 11 / 2007$ & $-6.65 \%$ & $-2.78 \%$ & $-1.20 \%$ \\
\hline 18 & $31 / 7 / 2003$ & $4.83 \%$ & $2.61 \%$ & $-1.77 \%$ & 71 & $31 / 12 / 2007$ & $1.39 \%$ & $4.59 \%$ & $1.46 \%$ \\
\hline 19 & $29 / 8 / 2003$ & $11.72 \%$ & $2.50 \%$ & $-1.29 \%$ & 72 & $31 / 1 / 2008$ & $-8.54 \%$ & $0.62 \%$ & $4.91 \%$ \\
\hline 20 & $30 / 9 / 2003$ & $7.83 \%$ & $-4.51 \%$ & $-0.41 \%$ & 73 & $29 / 2 / 2008$ & $7.88 \%$ & $8.79 \%$ & $-1.34 \%$ \\
\hline 21 & $31 / 10 / 2003$ & $10.46 \%$ & $3.77 \%$ & $-2.87 \%$ & 74 & $31 / 3 / 2008$ & $-2.36 \%$ & $-6.84 \%$ & $-0.06 \%$ \\
\hline 22 & $28 / 11 / 2003$ & $1.25 \%$ & $2.54 \%$ & $-2.68 \%$ & 75 & $30 / 4 / 2008$ & $3.02 \%$ & $5.20 \%$ & $-1.15 \%$ \\
\hline 23 & $31 / 12 / 2003$ & $19.58 \%$ & $4.94 \%$ & $0.20 \%$ & 76 & $30 / 5 / 2008$ & $0.35 \%$ & $6.91 \%$ & $-1.85 \%$ \\
\hline 24 & $30 / 1 / 2004$ & $-9.44 \%$ & $1.69 \%$ & $1.24 \%$ & 77 & $30 / 6 / 2008$ & $-7.73 \%$ & $11.84 \%$ & $-3.44 \%$ \\
\hline 25 & $27 / 2 / 2004$ & $2.51 \%$ & $8.49 \%$ & $1.11 \%$ & 78 & $31 / 7 / 2008$ & $-12.00 \%$ & $-9.10 \%$ & $4.48 \%$ \\
\hline 26 & $31 / 3 / 2004$ & $-8.59 \%$ & $2.13 \%$ & $1.64 \%$ & 79 & $29 / 8 / 2008$ & $2.08 \%$ & $-4.94 \%$ & $3.70 \%$ \\
\hline 27 & $30 / 4 / 2004$ & $0.86 \%$ & $2.27 \%$ & $-2.58 \%$ & 80 & $30 / 9 / 2008$ & $-12.19 \%$ & $-14.38 \%$ & $0.60 \%$ \\
\hline 28 & $31 / 5 / 2004$ & $-0.96 \%$ & $4.11 \%$ & $-0.06 \%$ & 81 & $31 / 10 / 2008$ & $-30.10 \%$ & $-22.27 \%$ & $3.57 \%$ \\
\hline 29 & $30 / 6 / 2004$ & $0.94 \%$ & $-4.16 \%$ & $-0.75 \%$ & 82 & $28 / 11 / 2008$ & $-3.37 \%$ & $-9.97 \%$ & $1.04 \%$ \\
\hline 30 & $30 / 7 / 2004$ & $-1.54 \%$ & $6.01 \%$ & $0.09 \%$ & 83 & $31 / 12 / 2008$ & $12.14 \%$ & $-8.98 \%$ & $7.50 \%$ \\
\hline 31 & $31 / 8 / 2004$ & $-1.23 \%$ & $1.41 \%$ & $1.03 \%$ & 84 & $30 / 1 / 2009$ & $-2.69 \%$ & $-4.47 \%$ & $-3.82 \%$ \\
\hline 32 & $30 / 9 / 2004$ & $3.40 \%$ & $6.70 \%$ & $0.86 \%$ & 85 & $27 / 2 / 2009$ & $-1.02 \%$ & $-0.53 \%$ & $-1.37 \%$ \\
\hline 33 & $29 / 10 / 2004$ & $-2.54 \%$ & $0.78 \%$ & $-0.29 \%$ & 86 & $31 / 3 / 2009$ & $1.03 \%$ & $2.98 \%$ & $1.70 \%$ \\
\hline 34 & $30 / 11 / 2004$ & $4.67 \%$ & $-4.91 \%$ & $-0.25 \%$ & 87 & $30 / 4 / 2009$ & $15.69 \%$ & $1.12 \%$ & $2.22 \%$ \\
\hline 35 & $31 / 12 / 2004$ & $1.82 \%$ & $-6.07 \%$ & $0.86 \%$ & 88 & 29/5/2009 & $14.42 \%$ & $13.52 \%$ & $-3.63 \%$ \\
\hline 36 & $31 / 1 / 2005$ & $5.11 \%$ & $2.17 \%$ & $0.12 \%$ & 89 & $30 / 6 / 2009$ & $6.64 \%$ & $-2.12 \%$ & $0.55 \%$ \\
\hline 37 & $28 / 2 / 2005$ & $5.72 \%$ & $6.49 \%$ & $0.73 \%$ & 90 & $31 / 7 / 2009$ & $4.43 \%$ & $2.24 \%$ & $0.82 \%$ \\
\hline 38 & $31 / 3 / 2005$ & $-6.69 \%$ & $5.70 \%$ & $1.63 \%$ & 91 & $31 / 8 / 2009$ & $5.50 \%$ & $-1.46 \%$ & $-0.03 \%$ \\
\hline 39 & $29 / 4 / 2005$ & $-2.31 \%$ & $-5.72 \%$ & $1.44 \%$ & 92 & $30 / 9 / 2009$ & $10.38 \%$ & $-1.27 \%$ & $-0.96 \%$ \\
\hline 40 & $31 / 5 / 2005$ & $1.48 \%$ & $3.08 \%$ & $0.13 \%$ & 93 & $30 / 10 / 2009$ & $-4.40 \%$ & $5.84 \%$ & $-0.76 \%$ \\
\hline 41 & $30 / 6 / 2005$ & $1.21 \%$ & $4.16 \%$ & $1.34 \%$ & 94 & $30 / 11 / 2009$ & $0.63 \%$ & $3.64 \%$ & $0.89 \%$ \\
\hline 42 & $29 / 7 / 2005$ & $0.03 \%$ & $4.49 \%$ & $-3.00 \%$ & 95 & $31 / 12 / 2009$ & $6.62 \%$ & $1.08 \%$ & $0.32 \%$ \\
\hline 43 & $31 / 8 / 2005$ & $4.10 \%$ & $4.39 \%$ & $-1.35 \%$ & 96 & $31 / 1 / 2010$ & $-5.17 \%$ & $-8.40 \%$ & $1.59 \%$ \\
\hline 44 & $30 / 9 / 2005$ & $3.86 \%$ & $0.44 \%$ & $-1.55 \%$ & 97 & $28 / 2 / 2010$ & $3.56 \%$ & $5.01 \%$ & $0.46 \%$ \\
\hline 45 & $31 / 10 / 2005$ & $-5.54 \%$ & $-5.79 \%$ & $-5.17 \%$ & 98 & $31 / 3 / 2010$ & $9.23 \%$ & $-1.53 \%$ & $0.17 \%$ \\
\hline 46 & $30 / 11 / 2005$ & $-2.10 \%$ & $0.17 \%$ & $2.50 \%$ & 99 & $30 / 4 / 2010$ & $-3.11 \%$ & $2.97 \%$ & $2.08 \%$ \\
\hline 47 & $30 / 12 / 2005$ & $6.89 \%$ & $4.70 \%$ & $3.26 \%$ & 100 & $31 / 5 / 2010$ & $-1.71 \%$ & $-10.00 \%$ & $1.06 \%$ \\
\hline 48 & $31 / 1 / 2006$ & $6.96 \%$ & $1.58 \%$ & $0.37 \%$ & 101 & $30 / 6 / 2010$ & $6.25 \%$ & $-0.17 \%$ & $0.88 \%$ \\
\hline 49 & $28 / 2 / 2006$ & $-2.37 \%$ & $-5.12 \%$ & $1.10 \%$ & 102 & $31 / 7 / 2010$ & $7.34 \%$ & $7.24 \%$ & $-0.33 \%$ \\
\hline 50 & $31 / 3 / 2006$ & $0.31 \%$ & $2.62 \%$ & $-1.15 \%$ & 103 & $31 / 8 / 2010$ & $6.70 \%$ & $-5.71 \%$ & $2.33 \%$ \\
\hline 51 & $28 / 4 / 2006$ & $5.56 \%$ & $2.43 \%$ & $0.68 \%$ & 104 & $30 / 9 / 2010$ & $6.80 \%$ & $5.39 \%$ & $0.26 \%$ \\
\hline 52 & $31 / 5 / 2006$ & $-7.41 \%$ & $1.29 \%$ & $0.71 \%$ & 105 & $31 / 10 / 2010$ & $0.94 \%$ & $3.31 \%$ & $-0.37 \%$ \\
\hline 53 & $30 / 6 / 2006$ & $-4.41 \%$ & $-0.36 \%$ & $-0.45 \%$ & 106 & $30 / 11 / 2010$ & $2.10 \%$ & $0.60 \%$ & $-1.53 \%$ \\
\hline
\end{tabular}


Table 2. Statistics of SET Total Return Index, Rogers International Commodity Index and ThaiBMA Government Bond Total Return Index during the year 2002-Nov 2010.

\begin{tabular}{|c|c|c|c|c|}
\hline & & \multicolumn{3}{|c|}{ MONTHLY RETURN } \\
\hline & SET TRI & $\mathbf{R I C I}^{\circledR}$ & BOND TRI \\
\hline \multicolumn{2}{|l|}{ Mean } & $1.57 \%$ & $0.75 \%$ & $0.41 \%$ \\
\hline \multicolumn{2}{|c|}{ Standard Deviation } & $6.76 \%$ & $5.46 \%$ & $1.90 \%$ \\
\hline \multicolumn{2}{|l|}{ Max } & $19.58 \%$ & $13.52 \%$ & $7.50 \%$ \\
\hline \multicolumn{2}{|l|}{ Min } & $-30.10 \%$ & $-22.27 \%$ & $-5.17 \%$ \\
\hline \multicolumn{5}{|c|}{ CORRELATION MATRIX } \\
\hline \multicolumn{3}{|c|}{ SET TRI } & RICI $^{\circledR}$ & BOND TRI \\
\hline \multirow{2}{*}{$\begin{array}{c}\text { SET TRI } \\
\text { RICI }^{\circledR}\end{array}$} & \multicolumn{2}{|c|}{1.0000} & & \\
\hline & \multicolumn{2}{|c|}{0.3771} & 1.0000 & \\
\hline BOND TRI & \multicolumn{2}{|c|}{-0.1160} & -0.2820 & 1.0000 \\
\hline
\end{tabular}

cordingly until the amount of investment in SET TRI, RICI ${ }^{\circledR}$ and BOND TRI at the end of the month 60 , which is equal to $45.129,78.084$ and 62.720 is obtained as shown in Table 3. For the reason that in randomization, the rate of return of SET

TRI, RICI ${ }^{\circledR}$ and BOND TRI in the same month has been used, the outcome of Bootstrapping Simulation run therefore has already taken into account the relationship among SET TRI, RICI ${ }^{\circledR}$ and BOND TRI rate of return, which is readily reflected in the correlation coefficients of this simulation run.

Step 4: Calculate the investment rate of return using the "Rate" function of Excel to determine the monthly rate of return. Given NPER $=60$ (60 month investment), PMT $=-3$ (investment of 3 Baht each month, comprising 1 Baht investment in SET TRI, another 1 Baht investment in RICI ${ }^{\circledR}$ and another 1 Baht in $\mathrm{BOND}$ TRI), $\mathrm{PV}=0, \mathrm{FV}=185.933$ (the total amount of investment in SET TRI, RICI ${ }^{\circledR}$ and BOND TRI at the end of month 60), and TYPE $=1$ (as investment starts at the beginning of each month); the resulting return on investment of $0.106 \%$ per month or $1.272 \%$ per year is obtained.

Step 5: Repeat step 1 through 4 up to 10000 rounds to find the distribution of investment portfolio rate of return. Figure 1 shows the distribution of investment portfolio rate of return from monthly investment of 1 Baht each in SET TRI, RICI ${ }^{\circledR}$ and BOND TRI, which can be described as a portfolio of an equal weight in common stocks, commodities and government bonds within the 5 years investment period.

Figure 1 shows that the investment portfolio has average annual return and standard deviation of $10.75 \%$ and $6.56 \%$ respectively, while the maximum and minimum annual rate of return equals $32.89 \%$ and
$-15.33 \%$, respectively. In addition, Figure $\mathbf{1}$ also shows that the VaR (Value at Risk) at $5 \%$ is $-0.08 \%$ (see the value at $5 \%$ Percentile); which means that the investment portfolio has a $5 \%$ chance that its annual return will be equal to or less than $-0.08 \%$, or in other words, the portfolio has a $95 \%$ chance that its annual return will be more than $-0.08 \%$.

\section{Results of the Study}

\subsection{Effects of Time Diversification}

Table 4 shows the statistics obtained as a result of the Bootstrapping Simulation runs based on monthly investment of $1 \mathrm{Baht}$ and various lengths of pre-retirement investment period using the $100 \%$ SET TRI investment scenario, $100 \%$ RICI ${ }^{\circledR}$ investment scenario and the $100 \%$ BOND TRI investment scenario. In the SET TRI investment scenario, in case we invested 1 Baht every month on SET TRI with 5 years period of pre-retirement investment, Table 4 shows that the investment portfolio offered $14.74 \%$ average rate of return with standard deviation of $12.32 \%$ and coefficient of variation of 0.836 ; which reflects that $0.836 \%$ of the variance (risk), as measured from standard deviation, has to be traded for every $1 \%$ of the average rate of return thus received. Furthermore, Table 4 shows the VaR value at $5 \%$ of this portfolio as $-6.79 \%$. The Table presents the outcome when the length of pre-retirement investment period increased from 5 years to 30 years. As the length of pre-retirement investment period increased by 5 years from 5 to 10 years, the average annual rate of return of the investment portfolio on SET TRI decreased by $0.43 \%$ from $14.74 \%$ to $14.31 \%$, but the risk measured from standard deviation decreased by as much as $3.51 \%$ from $12.32 \%$ to $8.81 \%$, resulting in the decrease of coefficient of variation from 0.836 to 0.616 . This reflects the time diversification phenomenon; that is, when the length of investment period increases, the risk is reduced in higher proportion than the rate of return is. In addition, the VaR value at $5 \%$ also increased from $-6.79 \%$ to $-0.92 \%$, which reveals that in case the length of pre-retirement investment period is 5 years, there is a $95 \%$ chance that the rate of return of the investment portfolio on SET TRI becomes greater than $-6.79 \%$; but if the length of pre-retirement investment period is increased to 10 years, there is a $95 \%$ chance that the rate of return of this investment portfolio becomes greater than $-0.92 \%$. This indicates that the risk of loss becomes lower with the increase in the length of investment period. Table 4 confirms the effect of time diversification where the duration extended from 5 years to 30 years, the average annual rate of return of the investment portfolio on SET TRI 
Table 3. Example of Bootstrapping Simulation run.

\begin{tabular}{|c|c|c|c|c|c|c|c|}
\hline \multirow{2}{*}{ Month } & \multirow{2}{*}{ Code } & \multicolumn{3}{|c|}{ Monthly Rate of Return } & \multicolumn{2}{|c|}{ Monthly Amount } & \multirow[b]{2}{*}{ BOND TRI } \\
\hline & & SET TRI & RICI ${ }^{\circledR}$ & BOND TRI & SET TRI & RICI ${ }^{\circledR}$ & \\
\hline 0 & & & & & 1.000 & 1.000 & 1.000 \\
\hline 1 & 33 & $-2.54 \%$ & $0.78 \%$ & $-0.29 \%$ & 1.975 & 2.008 & 1.997 \\
\hline 2 & 28 & $-0.96 \%$ & $4.11 \%$ & $-0.06 \%$ & 2.956 & 3.090 & 2.996 \\
\hline 3 & 1 & $9.33 \%$ & $3.47 \%$ & $-0.69 \%$ & 4.231 & 4.198 & 3.975 \\
\hline 4 & 26 & $-8.59 \%$ & $2.13 \%$ & $1.64 \%$ & 4.868 & 5.287 & 5.040 \\
\hline 5 & 54 & $1.98 \%$ & $0.70 \%$ & $1.18 \%$ & 5.964 & 6.324 & 6.100 \\
\hline 6 & 38 & $-6.69 \%$ & $5.70 \%$ & $1.63 \%$ & 6.565 & 7.685 & 7.199 \\
\hline 7 & 81 & $-30.10 \%$ & $-22.27 \%$ & $3.57 \%$ & 5.589 & 6.973 & 8.456 \\
\hline 8 & 25 & $2.51 \%$ & $8.49 \%$ & $1.11 \%$ & 6.730 & 8.566 & 9.550 \\
\hline 9 & 88 & $14.42 \%$ & $13.52 \%$ & $-3.63 \%$ & 8.700 & 10.724 & 10.204 \\
\hline 10 & 14 & $1.99 \%$ & $-6.81 \%$ & $-0.34 \%$ & 9.874 & 10.993 & 11.169 \\
\hline 11 & 46 & $-2.10 \%$ & $0.17 \%$ & $2.50 \%$ & 10.666 & 12.012 & 12.448 \\
\hline 12 & 20 & $7.83 \%$ & $-4.51 \%$ & $-0.41 \%$ & 12.502 & 12.471 & 13.397 \\
\hline 13 & 11 & $-2.19 \%$ & $4.71 \%$ & $0.60 \%$ & 13.228 & 14.059 & 14.477 \\
\hline 14 & 8 & $-8.02 \%$ & $5.49 \%$ & $1.87 \%$ & 13.167 & 15.830 & 15.748 \\
\hline 15 & 10 & $2.23 \%$ & $0.90 \%$ & $1.45 \%$ & 14.460 & 16.972 & 16.976 \\
\hline 16 & 43 & $4.10 \%$ & $4.39 \%$ & $-1.35 \%$ & 16.053 & 18.717 & 17.748 \\
\hline 17 & 47 & $6.89 \%$ & $4.70 \%$ & $3.26 \%$ & 18.159 & 20.597 & 19.326 \\
\hline 18 & 28 & $-0.96 \%$ & $4.11 \%$ & $-0.06 \%$ & 18.985 & 22.443 & 20.314 \\
\hline 19 & 68 & $4.46 \%$ & $9.25 \%$ & $0.50 \%$ & 20.831 & 25.520 & 21.415 \\
\hline 20 & 3 & $0.60 \%$ & $-0.72 \%$ & $1.18 \%$ & 21.957 & 26.337 & 22.668 \\
\hline 21 & 30 & $-1.54 \%$ & $6.01 \%$ & $0.09 \%$ & 22.618 & 28.919 & 23.689 \\
\hline 22 & 81 & $-30.10 \%$ & $-22.27 \%$ & $3.57 \%$ & 16.811 & 23.479 & 25.535 \\
\hline 23 & 53 & $-4.41 \%$ & $-0.36 \%$ & $-0.45 \%$ & 17.070 & 24.395 & 26.421 \\
\hline 24 & 6 & $-3.36 \%$ & $1.77 \%$ & $1.71 \%$ & 17.497 & 25.827 & 27.872 \\
\hline 25 & 49 & $-2.37 \%$ & $-5.12 \%$ & $1.10 \%$ & 18.081 & 25.505 & 29.178 \\
\hline 26 & 28 & $-0.96 \%$ & $4.11 \%$ & $-0.06 \%$ & 18.908 & 27.554 & 30.160 \\
\hline 27 & 16 & $7.93 \%$ & $5.48 \%$ & $2.00 \%$ & 21.408 & 30.064 & 31.764 \\
\hline 28 & 65 & $5.35 \%$ & $2.68 \%$ & $-2.52 \%$ & 23.554 & 31.869 & 31.963 \\
\hline 29 & 19 & $11.72 \%$ & $2.50 \%$ & $-1.29 \%$ & 27.315 & 33.664 & 32.552 \\
\hline 30 & 38 & $-6.69 \%$ & $5.70 \%$ & $1.63 \%$ & 26.488 & 36.583 & 34.082 \\
\hline 31 & 70 & $-6.65 \%$ & $-2.78 \%$ & $-1.20 \%$ & 25.727 & 36.566 & 34.672 \\
\hline 32 & 77 & $-7.73 \%$ & $11.84 \%$ & $-3.44 \%$ & 24.738 & 41.894 & 34.478 \\
\hline 33 & 58 & $2.37 \%$ & $3.13 \%$ & $2.17 \%$ & 26.324 & 44.204 & 36.227 \\
\hline 34 & 13 & $-2.02 \%$ & $5.88 \%$ & $0.01 \%$ & 26.792 & 47.803 & 37.230 \\
\hline 35 & 2 & $1.21 \%$ & $10.52 \%$ & $-2.07 \%$ & 28.116 & 53.831 & 37.458 \\
\hline 36 & 28 & $-0.96 \%$ & $4.11 \%$ & $-0.06 \%$ & 28.846 & 57.044 & 38.435 \\
\hline 37 & 46 & $-2.10 \%$ & $0.17 \%$ & $2.50 \%$ & 29.240 & 58.144 & 40.395 \\
\hline 38 & 8 & $-8.02 \%$ & $5.49 \%$ & $1.87 \%$ & 27.894 & 62.334 & 42.150 \\
\hline 39 & 8 & $-8.02 \%$ & $5.49 \%$ & $1.87 \%$ & 26.657 & 66.754 & 43.938 \\
\hline 40 & 94 & $0.63 \%$ & $3.64 \%$ & $0.89 \%$ & 27.824 & 70.184 & 45.329 \\
\hline 41 & 60 & $-3.70 \%$ & $-1.99 \%$ & $3.53 \%$ & 27.795 & 69.785 & 47.927 \\
\hline 42 & 79 & $2.08 \%$ & $-4.94 \%$ & $3.70 \%$ & 29.374 & 67.339 & 50.699 \\
\hline 43 & 75 & $3.02 \%$ & $5.20 \%$ & $-1.15 \%$ & 31.262 & 71.843 & 51.118 \\
\hline 44 & 49 & $-2.37 \%$ & $-5.12 \%$ & $1.10 \%$ & 31.519 & 69.166 & 52.679 \\
\hline 45 & 58 & $2.37 \%$ & $3.13 \%$ & $2.17 \%$ & 33.266 & 72.330 & 54.824 \\
\hline 46 & 27 & $0.86 \%$ & $2.27 \%$ & $-2.58 \%$ & 34.551 & 74.968 & 54.412 \\
\hline 47 & 89 & $6.64 \%$ & $-2.12 \%$ & $0.55 \%$ & 37.844 & 74.381 & 55.712 \\
\hline 48 & 59 & $-7.96 \%$ & $-4.81 \%$ & $-2.41 \%$ & 35.831 & 71.806 & 55.370 \\
\hline 49 & 39 & $-2.31 \%$ & $-5.72 \%$ & $1.44 \%$ & 36.005 & 68.702 & 57.169 \\
\hline 50 & 5 & $-4.58 \%$ & $1.93 \%$ & $1.08 \%$ & 35.357 & 71.025 & 58.785 \\
\hline 51 & 10 & $2.23 \%$ & $0.90 \%$ & $1.45 \%$ & 37.145 & 72.661 & 60.638 \\
\hline 52 & 43 & $4.10 \%$ & $4.39 \%$ & $-1.35 \%$ & 39.668 & 76.852 & 60.822 \\
\hline 53 & 3 & $0.60 \%$ & $-0.72 \%$ & $1.18 \%$ & 40.907 & 77.300 & 62.540 \\
\hline 54 & 28 & $-0.96 \%$ & $4.11 \%$ & $-0.06 \%$ & 41.515 & 81.478 & 63.502 \\
\hline 55 & 21 & $10.46 \%$ & $3.77 \%$ & $-2.87 \%$ & 46.859 & 85.552 & 62.676 \\
\hline 56 & 22 & $1.25 \%$ & $2.54 \%$ & $-2.68 \%$ & 48.444 & 88.724 & 61.996 \\
\hline 57 & 19 & $11.72 \%$ & $2.50 \%$ & $-1.29 \%$ & 55.122 & 91.938 & 62.199 \\
\hline 58 & 80 & $-12.19 \%$ & $-14.38 \%$ & $0.60 \%$ & 49.400 & 79.717 & 63.571 \\
\hline 59 & 45 & $-5.54 \%$ & $-5.79 \%$ & $-5.17 \%$ & 47.663 & 76.103 & 61.287 \\
\hline 60 & 52 & $-7.41 \%$ & $1.29 \%$ & $0.71 \%$ & 45.129 & 78.084 & 62.720 \\
\hline
\end{tabular}




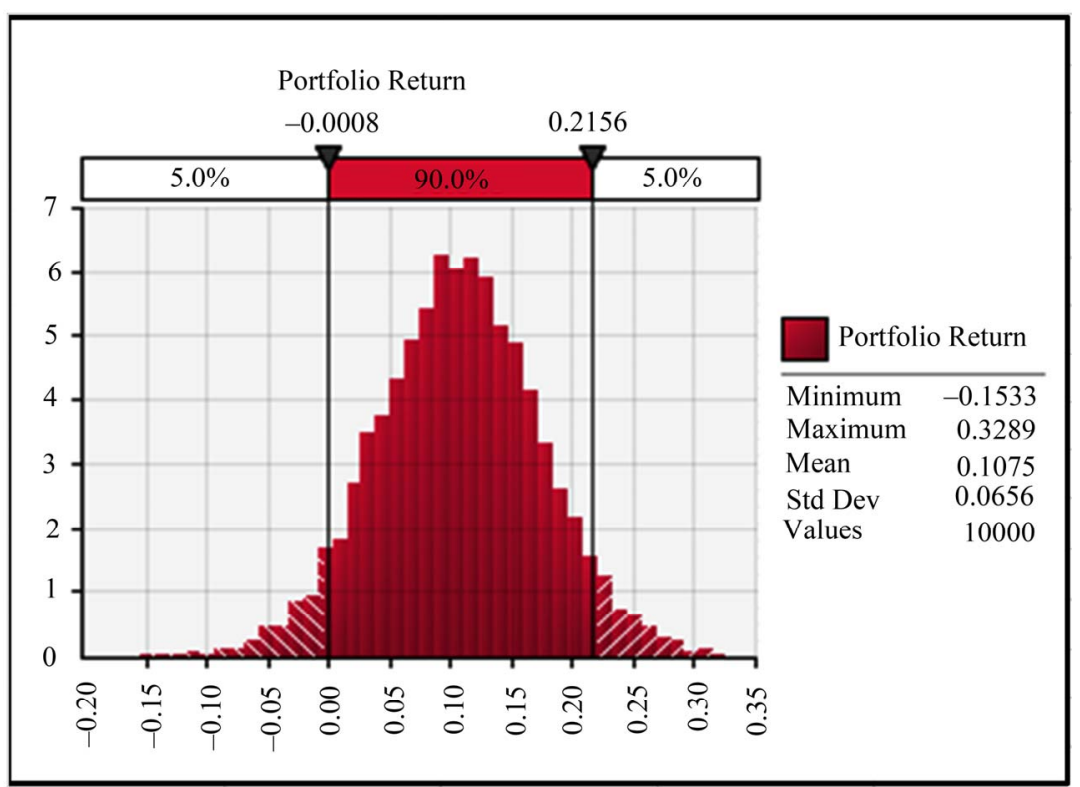

\begin{tabular}{cc}
\hline & Simulation Summary Information \\
\hline Workbook Name & Portfolio Optimization Results new version.xls \\
Number of Simulations & 1 \\
Number of Tterations & 10000 \\
Number of Inputs & 60 \\
Number of outputs & 1 \\
Sampling Type & Latin Hypercube \\
Simulation Start Time & $1 / 28 / 1118: 46: 53$ \\
Simulation Duration & $00: 00: 20$ \\
Random \# Generator & Mersenne Twister \\
Random Seed & 1654279895 \\
\hline
\end{tabular}

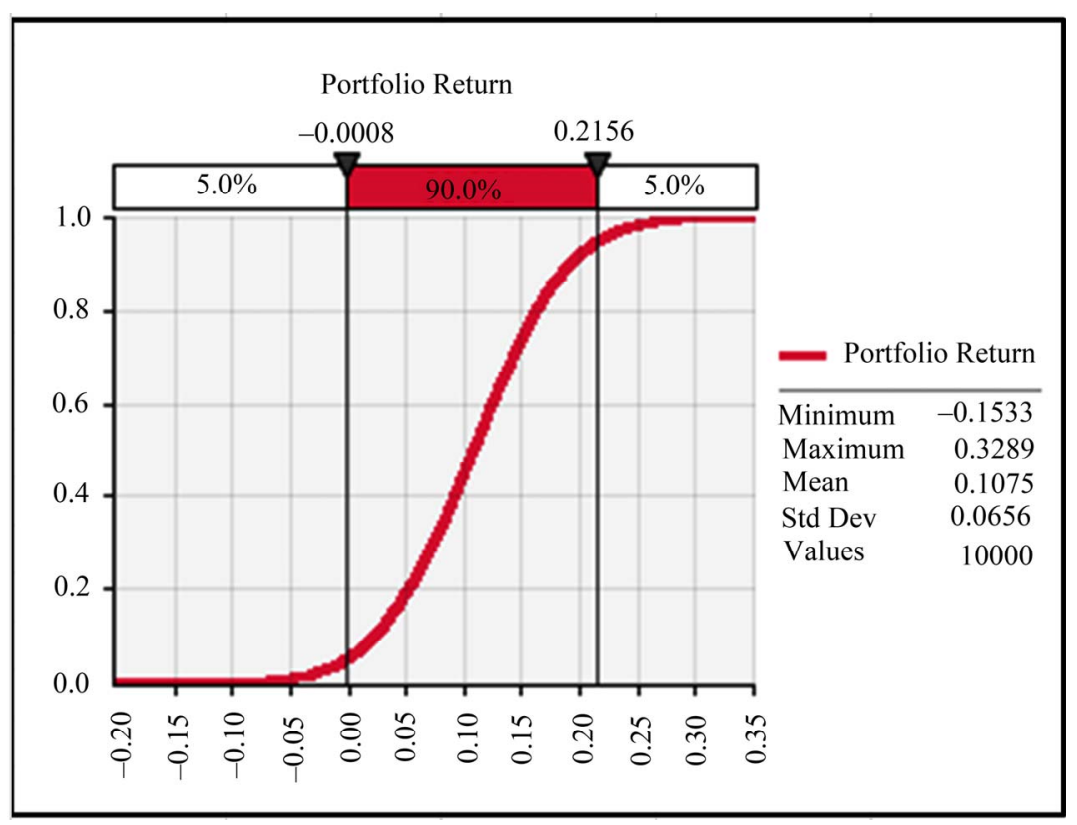




\begin{tabular}{|c|c|c|c|}
\hline \multicolumn{4}{|c|}{ Summary Statistics for Portfolio Return } \\
\hline \multicolumn{2}{|c|}{ Statistics } & \multicolumn{2}{|c|}{ Percentile } \\
\hline Minimum & $-15.33 \%$ & $5 \%$ & $-0.08 \%$ \\
\hline Maximum & $32.89 \%$ & $10 \%$ & $2.33 \%$ \\
\hline Mean & $10.75 \%$ & $15 \%$ & $3.88 \%$ \\
\hline Std Dev & $6.56 \%$ & $20 \%$ & $5.19 \%$ \\
\hline Variance & 0.004303236 & $25 \%$ & $6.34 \%$ \\
\hline Skewness & -0.011767783 & $30 \%$ & $7.38 \%$ \\
\hline Kurtosis & 2.976404013 & $35 \%$ & $8.30 \%$ \\
\hline Median & $10.75 \%$ & $40 \%$ & $9.16 \%$ \\
\hline Mode & $11.84 \%$ & $45 \%$ & $9.96 \%$ \\
\hline Left X & $-0.08 \%$ & $50 \%$ & $10.75 \%$ \\
\hline Left P & $5 \%$ & $55 \%$ & $11.60 \%$ \\
\hline Right X & $21.56 \%$ & $60 \%$ & $12.40 \%$ \\
\hline Right P & $95 \%$ & $65 \%$ & $13.27 \%$ \\
\hline Diff $X$ & $21.64 \%$ & $70 \%$ & $14.22 \%$ \\
\hline Diff P & $90 \%$ & $75 \%$ & $15.17 \%$ \\
\hline \# Errors & $0 \%$ & $80 \%$ & $16.20 \%$ \\
\hline Filter Min & off & $85 \%$ & $17.48 \%$ \\
\hline Filter Max & off & $90 \%$ & $19.17 \%$ \\
\hline \# Filtered & $0 \%$ & $95 \%$ & $21.56 \%$ \\
\hline
\end{tabular}

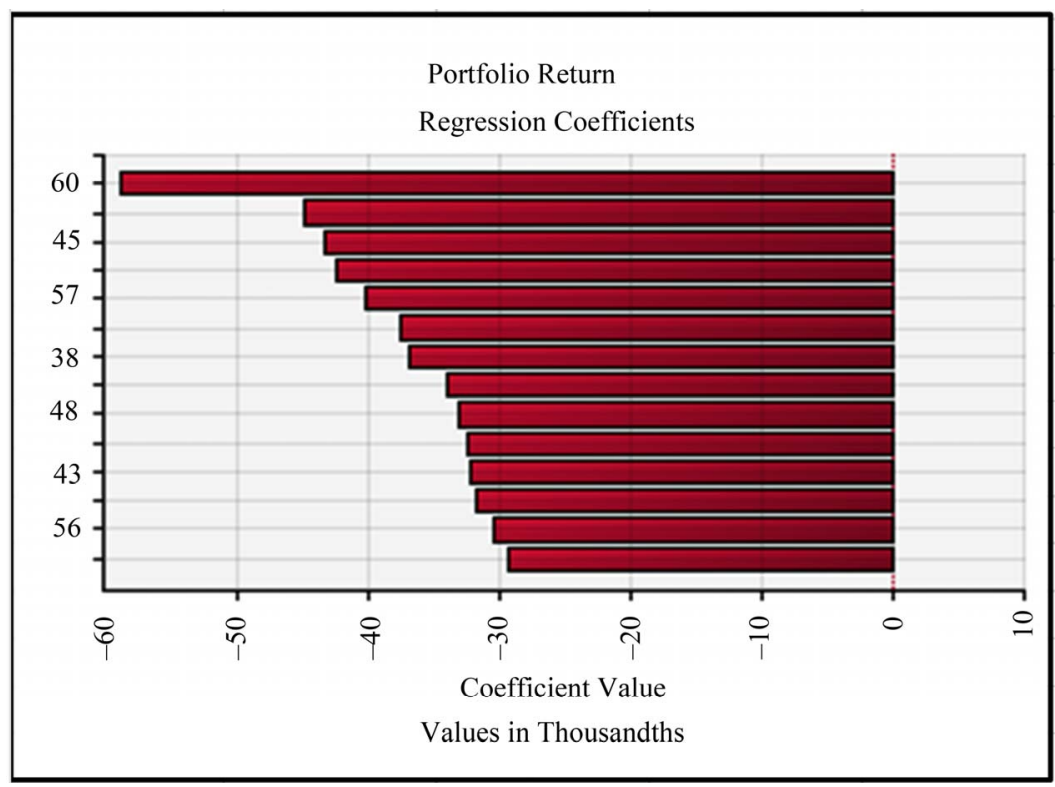

\begin{tabular}{cccc}
\hline \multicolumn{5}{c}{ Regression and Rank Information for Portfolio Return } \\
\hline Rank & Name & Regr & Corr \\
1 & 60 & -0.059 & -0.053 \\
2 & 36 & -0.045 & -0.046 \\
3 & 45 & -0.043 & -0.046 \\
4 & 52 & -0.043 & -0.043 \\
5 & 57 & -0.040 & -0.041 \\
6 & 34 & -0.038 & -0.036 \\
7 & 38 & -0.037 & -0.039 \\
8 & 50 & -0.034 & -0.037 \\
9 & 48 & -0.033 & -0.028 \\
10 & 44 & -0.032 & -0.028 \\
11 & 43 & -0.032 & -0.033 \\
12 & 31 & -0.032 & -0.033 \\
13 & 56 & -0.030 & -0.033 \\
14 & 32 & -0.029 & -0.033 \\
\hline
\end{tabular}

Figure 1. Distribution of investment portfolio rate of return generated through Bootstrapping Simulation. 
Table 4. Statistics from a Bootstrapping Simulation run with monthly investment of 1 Baht in SET TRI, RICI ${ }^{\circledR}$ and BOND TRI at various lengths of pre-investment period.

\begin{tabular}{|c|c|c|c|c|c|c|c|}
\hline & & \multicolumn{6}{|c|}{ Lengths of Pre-retirement Investment Period } \\
\hline & & 5 yrs & $10 \mathrm{yrs}$ & 15 yrs & $20 \mathrm{yrs}$ & $25 \mathrm{yrs}$ & 30 yrs \\
\hline & SET TRI & $14.74 \%$ & $14.31 \%$ & $14.23 \%$ & $14.19 \%$ & $14.18 \%$ & $14.15 \%$ \\
\hline \multirow[t]{3}{*}{ Average Annual Rate of Return } & $\mathrm{RICI}^{\circledR}$ & $9.18 \%$ & $8.75 \%$ & $8.67 \%$ & $8.65 \%$ & $8.62 \%$ & $8.61 \%$ \\
\hline & BOND TRI & $5.01 \%$ & $4.55 \%$ & $4.48 \%$ & $4.46 \%$ & $4.45 \%$ & $4.44 \%$ \\
\hline & SET TRI & $12.32 \%$ & $8.81 \%$ & $6.91 \%$ & $6.08 \%$ & $5.26 \%$ & $4.78 \%$ \\
\hline \multirow[t]{3}{*}{ Standard Deviation } & $\mathrm{RICI}^{\circledR}$ & $9.70 \%$ & $6.84 \%$ & $5.54 \%$ & $4.73 \%$ & $4.22 \%$ & $3.85 \%$ \\
\hline & BOND TRI & $3.40 \%$ & $2.41 \%$ & $1.96 \%$ & $1.71 \%$ & $1.52 \%$ & $1.37 \%$ \\
\hline & SET TRI & 0.836 & 0.616 & 0.486 & 0.428 & 0.371 & 0.338 \\
\hline \multirow[t]{3}{*}{ Coefficient of Variation } & $\mathrm{RICI}^{\circledR}$ & 1.057 & 0.782 & 0.639 & 0.547 & 0.490 & 0.447 \\
\hline & BOND TRI & 0.679 & 0.530 & 0.438 & 0.383 & 0.342 & 0.309 \\
\hline & SET TRI & $-6.79 \%$ & $-0.92 \%$ & $2.32 \%$ & $3.98 \%$ & $5.37 \%$ & $6.12 \%$ \\
\hline \multirow[t]{2}{*}{ Value at Risk at 5\% } & $\mathrm{RICI}^{\circledR}$ & $-7.20 \%$ & $-2.70 \%$ & $-0.73 \%$ & $0.60 \%$ & $1.55 \%$ & $2.19 \%$ \\
\hline & BOND TRI & $-0.55 \%$ & $0.53 \%$ & $1.18 \%$ & $1.61 \%$ & $1.91 \%$ & $2.19 \%$ \\
\hline
\end{tabular}

decreased from $14.74 \%$ to $14.15 \%$ while the risk measured from standard deviation decreased from $12.32 \%$ to $4.78 \%$, and the coefficient of variation from 0.836 to 0.338 . The VaR value at $5 \%$ increased from $-6.79 \%$ to $6.12 \%$ which means that in case the length of pre-retirement investment period is 30 years, we have as much as $95 \%$ chance that the rate of return of the investment portfolio on SET TRI becomes greater than $6.12 \%$, which in turn reflects the lower risk of loss.

Upon considering the $100 \%$ investment portfolio on RICI ${ }^{\circledR}$ and BOND TRI, we experience the same time diversification effect as in the case of investment portfolio on SET TRI; that is, when the duration extended from 5 years to 30 years, the average annual rate of return de-creased from $9.18 \%$ to $8.61 \%$ for the investment portfolio on RICI® and from $5.01 \%$ to $4.44 \%$ for the investment portfolio on BOND TRI while the risk measured from standard deviation decreased from $9.70 \%$ to $3.85 \%$ for the investment portfolio on RICI ${ }^{\circledR}$ and from $3.40 \%$ to $1.37 \%$ for the investment portfolio on BOND TRI and the coefficient of variation from 1.057 to 0.447 for the investment portfolio on RICI ${ }^{\circledR}$ and from 0.679 to 0.309 for the investment portfolio on BOND TRI. The VaR value at $5 \%$ increased from $-7.20 \%$ to $2.19 \%$ for the investment portfolio on $\mathrm{RICI}{ }^{\circledR}$ and from $-0.55 \%$ to $2.19 \%$ for the investment portfolio on BOND TRI.

\subsection{Determination of the Target Rate of Return for Retirement}

This section involves the determination of the target rate of return to achieve the retirement goal being laid out, where the target rate of return varies with the length of investment period, the amount of pre-retirement investment, the life span, and the amount required for spending after retirement.

In the case of Jenny who is currently 30 years old and plans to retire at the age of 60 ; Jenny has a pre-retirement investment period of 30 years. According to the World Health Organization, the average age of Thai men and Thai women are 69 years and 75 years respectively. Therefore, it can be expected that Jenny shall be living another 15 years after retirement. Jenny intends to invest for her retirement the amount of 10,000 Baht at the beginning of each month for the next 30 years up to retirement. She wishes to earn for post-retirement spending 5 times the amount of pre-retirement investment on monthly basis. In her case the amount is 50,000 Baht. In order to get a conservative estimate, this study was based on the assumption that the post-retirement rate of return was zero. Therefore, Jenny needs to have the total amount of 9,000,000 Baht upon retirement (50,000 Baht per month $\times 15$ years $\times 12$ months per year) in order to be able to withdraw from the sum the monthly amount of 50,000 Baht for 15 years. Based on this assumption, Jenny's pre-retirement investment portfolio shall have the target rate of return of $0.45 \%$ per month or $5.40 \%$ per year.

Table 5 shows the target rate of return under various assumptions concerning the length of pre-retirement investment period and the required amount for post-retirement spending. In the case of Jenny, the target rate of return is shown on the top left hand corner of the table, which is the case where the length of pre-retirement in 
Table 5. Target rate of return under the hypothesis of various combinations of the lengths of pre-retirement investment period and the required amounts for post-retirement spending.

\begin{tabular}{ccccccc}
\hline $\begin{array}{c}\text { Lengths of } \\
\text { pre-retirement in- } \\
\text { vestment period }\end{array}$ & \multicolumn{5}{c}{$\begin{array}{c}\text { Required monthly amounts for 15 years post-retirement spending } \\
\text { calculated as number of times of monthly pre-retirement investment }\end{array}$} \\
\cline { 2 - 6 } & 5 times & 10 times & 15 times & 20 times & 25 times & 30 times \\
\hline 30 years & $5.40 \%$ & $8.88 \%$ & $10.78 \%$ & $12.09 \%$ & $13.08 \%$ & $13.88 \%$ \\
25 years & $7.62 \%$ & $11.70 \%$ & $13.95 \%$ & $15.49 \%$ & $16.66 \%$ & $17.61 \%$ \\
20 years & $11.22 \%$ & $16.20 \%$ & $18.95 \%$ & $20.85 \%$ & $22.31 \%$ & $23.48 \%$ \\
15 years & $17.80 \%$ & $24.25 \%$ & $27.86 \%$ & $30.37 \%$ & $32.28 \%$ & $33.83 \%$ \\
\hline
\end{tabular}

vestment period is 30 years and the required monthly amount for post-retirement spending is 5 times the monthly amount invested before retirement. From Table 3 it is found that the target rate of return of the pre-retirement investment portfolio increases with the increase in the amount required for post-retirement spending and/or the decrease in the length of pre-retirement investment period. If the length of your pre-retirement investment period is merely 15 years, but the required amount for post-retirement spending is 30 times the amount invested before retirement; in such case the retirement investment portfolio needs to have the target rate of return as high as $33.83 \%$ per year.

\subsection{Optimal Investment Portfolio to Achieve the Target Rate of Return at Minimum}

Researches on portfolio optimization have mostly conducted the optimization by calculating the average rate of return and standard deviation based on historical common stock and government bond data, and using the value thus obtained in a model to determine the optimal proportion of investment portfolio to achieve the goal of retirement under lowest risk, where the risk is measured from the standard deviation value. The Solver function of Excel can be used for solving such problem. However, the said optimization has been under the hypothesis that the future rate of return and standard deviation of the common stocks and government bonds shall be equal to the average rate of return and standard deviation of the historical common stocks and government bonds, without regard to the uncertainty of the future rate of return. In our study, the RISKOptimizer program is employed to take into account the uncertainty of the future rate of return in conducting portfolio optimization; which will yield more reasonable results. The RISKOptimizer program includes simulation and optimization techniques to allow optimization of the problems having variables with uncertainty in the model by applying the genetic algorithms based optimization technique and Monte Carlo simulation technique. RISKOptimization can be used to analyze the problems and reach a proper answer which could not be found through the use of normal linear and non-linear optimization programs such as the Solver function of Excel.

An optimal investment portfolio for retirement can be obtained by solving the optimization problem as follows:

$$
\operatorname{Min}_{w_{B O N D T R I}, w_{\text {SETTRI }}, w_{R I C I}} \sigma_{p}
$$

Subject to the following limitations:

$$
\begin{aligned}
& \mu_{p} \geq R_{\text {Targ } e t} \\
& w_{\text {BONDTRI }}+w_{\text {SETTRI }}+w_{\text {RICI }}=1 \\
& w_{\text {BONDTRI }} \geq 0 \\
& w_{\text {SETTRI }} \geq 0 \\
& w_{\text {RICI }} \geq 0
\end{aligned}
$$

where:

$\sigma_{p}=$ standard deviation of investment portfolio rate of return

$\mu_{\mathrm{p}}=$ average rate of return of the investment portfolio

$\mathrm{R}_{\text {Target }}=$ target rate of return for retirement

$\mathrm{w}_{\mathrm{BONDTRI}}=$ optimal proportion of BOND TRI

$\mathrm{W}_{\text {SETTRI }}=$ optimal proportion of SET TRI

$\mathrm{W}_{\mathrm{RICI}}=$ optimal proportion of RICI ${ }^{\circledR}$

From the foregoing explanation, this study employs genetic algorithms based optimization feature of the RISKOptimizer program to analyze and find an optimal proportion of investment in SET TRI and BOND TRI in order to achieve the target rate of return for retirement at the lowest risk measured from the standard deviation value.

Table 6 shows the proportion of SET TRI, RICI ${ }^{\circledR}$, and BOND TRI in an optimal investment portfolio for various combinations of the lengths of pre-retirement investment period and the required amounts for post-retirement spending. In the case of Jenny, where the pre-retirement investment period is 30 years and the required monthly amount for post-retirement spending is 5 times the monthly pre-retirement investment amount. Table 5 shows that the target rate of return of Jenny's investment portfolio is $5.40 \%$. Table 6 shows that the investment portfolio for retirement suitable for Jenny is the investment proportion of $0.59 \%$ in SET TRI, $6.02 \%$ in RICI ${ }^{\circledR}$, and $93.39 \%$ in BOND TRI, where the portfolio offers the average rate of return, as calculated by 
Table 6. Optimal investment portfolio for various combinations of the lengths of pre-retirement investment period and the required amounts for post-retirement spending.

\begin{tabular}{|c|c|c|c|c|c|c|c|}
\hline \multirow{2}{*}{$\begin{array}{c}\text { Lengths of } \\
\text { Pre-retirement } \\
\text { Investment } \\
\text { Period }\end{array}$} & & \multicolumn{6}{|c|}{$\begin{array}{l}\text { Required monthly amounts for } 15 \text { years post-retirement spending } \\
\text { calculated as number of times of monthly pre-retirement investment }\end{array}$} \\
\hline & & 5 times & 10 times & 15 times & 20 times & 25 times & 30 times \\
\hline \multirow[t]{8}{*}{30 years } & Target Rate of Return & $5.43 \%$ & $8.92 \%$ & $10.83 \%$ & $12.13 \%$ & $13.13 \%$ & $13.93 \%$ \\
\hline & Optimum Investment Proportion in SET TRI & $0.59 \%$ & $6.79 \%$ & $23.16 \%$ & $41.02 \%$ & $66.71 \%$ & $92.01 \%$ \\
\hline & Optimum Investment Proportion in $\mathrm{RICI}^{\circledR}$ & $6.02 \%$ & $48.46 \%$ & $48.52 \%$ & $48.23 \%$ & $22.54 \%$ & $4.78 \%$ \\
\hline & Optimum Investment Proportion in BOND TRI & $93.39 \%$ & $44.76 \%$ & $28.32 \%$ & $10.76 \%$ & $10.76 \%$ & $3.21 \%$ \\
\hline & Average rate of return & $5.45 \%$ & $8.93 \%$ & $10.88 \%$ & $12.16 \%$ & $13.16 \%$ & $13.94 \%$ \\
\hline & Standard deviation & $1.26 \%$ & $2.81 \%$ & $3.51 \%$ & $3.91 \%$ & $4.31 \%$ & $4.72 \%$ \\
\hline & VaR at $5 \%$ & $3.46 \%$ & $4.60 \%$ & $5.34 \%$ & $5.87 \%$ & $6.25 \%$ & $6.06 \%$ \\
\hline & Relative VarR at $5 \%$ & $-1.97 \%$ & $-4.32 \%$ & $-5.49 \%$ & $-6.26 \%$ & $-6.88 \%$ & $-7.87 \%$ \\
\hline \multirow[t]{8}{*}{25 years } & Target rate of return & $7.67 \%$ & $11.76 \%$ & $14.01 \%$ & $15.56 \%$ & $16.74 \%$ & $17.69 \%$ \\
\hline & Optimum investment proportion in SET TRI & $3.39 \%$ & $45.62 \%$ & $94.64 \%$ & \multirow{7}{*}{\multicolumn{3}{|c|}{ N/A }} \\
\hline & Optimum investment proportion in $\mathrm{RICI}^{\circledR}$ & $38.25 \%$ & $14.95 \%$ & $2.50 \%$ & & & \\
\hline & Optimum investment proportion in BOND TRI & $58.36 \%$ & $39.43 \%$ & $2.86 \%$ & & & \\
\hline & Average rate of return & $7.79 \%$ & $11.78 \%$ & $14.02 \%$ & & & \\
\hline & Standard deviation & $2.48 \%$ & $4.23 \%$ & $5.24 \%$ & & & \\
\hline & $\mathrm{VaR}$ at $5 \%$ & $4.12 \%$ & $5.24 \%$ & $5.22 \%$ & & & \\
\hline & Relative VarR at $5 \%$ & $-3.55 \%$ & $-6.52 \%$ & $-8.79 \%$ & & & \\
\hline \multirow[t]{8}{*}{20 years } & Target rate of return & $11.30 \%$ & $16.29 \%$ & $19.06 \%$ & $20.97 \%$ & $22.43 \%$ & $23.60 \%$ \\
\hline & Optimum investment proportion in SET TRI & $34.84 \%$ & \multirow{7}{*}{\multicolumn{5}{|c|}{$\mathrm{N} / \mathrm{A}$}} \\
\hline & Optimum investment proportion in $\mathrm{RICI}^{\circledR}$ & $41.37 \%$ & & & & & \\
\hline & Optimum investment proportion in BOND TRI & $23.79 \%$ & & & & & \\
\hline & Average rate of return & $11.32 \%$ & & & & & \\
\hline & Standard deviation & $4.33 \%$ & & & & & \\
\hline & VaR at $5 \%$ & $4.41 \%$ & & & & & \\
\hline & Relative VarR at $5 \%$ & $-6.89 \%$ & & & & & \\
\hline \multirow[t]{8}{*}{15 years } & Target rate of return & $17.95 \%$ & $24.44 \%$ & $28.07 \%$ & $30.58 \%$ & $32.51 \%$ & $34.07 \%$ \\
\hline & Optimum investment proportion in SET TRI & \multirow{7}{*}{\multicolumn{6}{|c|}{ N/A }} \\
\hline & Optimum investment proportion in $\mathrm{RICI}^{\mathbb{R}}$ & & & & & & \\
\hline & Optimum investment proportion in BOND TRI & & & & & & \\
\hline & Average rate of return & & & & & & \\
\hline & Standard deviation & & & & & & \\
\hline & $\mathrm{VaR}$ at $5 \%$ & & & & & & \\
\hline & Relative VarR at $5 \%$ & & & & & & \\
\hline
\end{tabular}

Bootstrapping Simulation, of $5.45 \%$, which is higher than Jenny's target rate of return of $5.40 \%$. The lowest risk as measured from standard deviation is $1.26 \%$; while the $\mathrm{VaR}$ at $5 \%$ of the rate of return is $3.46 \%$, which means that there is a $5 \%$ chance that the portfolio will yield less than or equal to $3.46 \%$. When compared with the target rate of return of $5.43 \%$, the Relative VaR at $5 \%$, or the $\mathrm{VaR}$ at $5 \%$ of the investment portfolio as measured with respect to the target rate of return, is equal to $-1.94 \%(=3.46 \%-5.40 \%)$; which means that there is a $5 \%$ chance that this portfolio will yield less than the set target by the amount of $1.94 \%$ or more.

Taking into consideration the case where the pre-retirement investment period is equal to 30 years and the required monthly amount for post-retirement spending increases from 5 times to 30 times the monthly pre-re- 
tirement investment amount, Table 6 shows that the optimal investment proportion in SET TRI increases from $0.59 \%$ to $92.01 \%$, and the optimal investment proportion in BOND TRI decreases from $93.39 \%$ to $3.21 \%$. However, the optimal investment proportion in RICI ${ }^{\circledR}$ increases from $6.02 \%$ to $48.52 \%$ when the required monthly amount for post-retirement spending increases from 5 times to 15 times the monthly pre-retirement investment amount and decreases to $4.78 \%$ when the required monthly amount for post-retirement spending increases to 30 times the monthly pre-retirement investment amount. Regarding the risk measured from standard deviation, it increases from $1.26 \%$ to $4.72 \%$ and the risk measured from the Relative VaR at $5 \%$ of the rate of return is reduced from $-1.94 \%$ to $-7.82 \%$. For other lengths of pre-retirement investment period, the outcomes are similar to the case where the pre-retirement investment period is 30 years; that is, when the required monthly amount for post-retirement spending increases, the investment proportion in SET TRI in the optimal investment portfolio for retirement shall also increase, which results in higher risk of the investment portfolio.

In the case where the required monthly amount for post-retirement spending is equal to 5 times and the length of pre-retirement investment period is reduced from 30 years to 20 years, Table 6 shows that the optimal investment proportion in SET TRI increases from $0.59 \%$ to $34.84 \%$, and the optimal investment proportion in $\mathrm{RICI} \AA$ increases from $6.02 \%$ to $41.37 \%$, while the optimal investment proportion in BOND TRI decreases from $93.39 \%$ to $23.79 \%$. When considering the risk, standard deviation increases from $1.26 \%$ to $4.33 \%$ and the risk measured from the Relative $\mathrm{VaR}$ at $5 \%$ of the rate of return is reduced from $-1.94 \%$ to $-6.81 \%$. For other required monthly amounts for post-retirement spending, Table 6 demonstrates the benefit of time diversification; that is, while the pre-retirement investment period is longer, the risk of the investment portfolio for retirement is reduced. In addition, Table 6 also shows that the goal of required amount for post-retirement spending might not be achieved if the pre-retirement investment period is not long enough. For example, if the pre-retirement investment period is 25 years, but the required monthly amount for post-retirement spending is 20 times the monthly pre-retirement investment amount; in such case the portfolio is required to offer a target return of $15.49 \%$, which is not achievable even with $100 \%$ investment in SET TRI due to the fact that the average return of SET TRI is $14.18 \%$ as exhibited in Table 4 . In case the pre-retirement investment period is 20 years, the goal of having the monthly amount for post-retirement spending as large as 10 times the monthly pre-retirement investment amount is not achievable. While our pre-re- tirement investment period is reduced to only 15 years, we will not be able to reach the goal of having the monthly amount for post-retirement spending even as low as 5 times the monthly pre-retirement investment amount.

\section{Summary}

Through the use of Bootstrapping Simulation technique in modeling long-term rate of returns based on various combinations of the lengths of retirement investment period and the required amounts for post-retirement spending, this research was conducted to find an optimal proportion of common stocks, commodities and government bonds to achieve the target rate of return for retirement by minimizing investment portfolio risk measured from standard deviation value. Also included was risk analysis based on the Value at Risk concept for studying downside risk of the investment portfolio for retirement. The study has found that in an optimal portfolio for retirement, the proportion of investment in SET TRI becomes smaller, resulting in a corresponding decrease of portfolio risk, as the length of pre-retirement investment period increases and/or the required amount for post-retirement spending decreases. Furthermore, this study reveals that the longer the period of pre-retirement investment, the lower is the risk of the retirement portfolio due to the effect of time diversification, and the larger is the receivable amount for post-retirement spending.

This research reflects that the government should conduct a publicity campaign to make the Thai society perceive the importance of long-term investment, by encouraging working-age citizens to invest for retirement early so as to have longer pre-retirement investment period, which shall in turn reduce the risk of retirement portfolio to acceptable level, and to make available the anticipated amount for post-retirement spending. If this could be realized, the government burden with regard to provision of welfare for retired citizens in the future shall be well relieved.

\section{References}

[1] R. A. Levy, "Stocks, Bonds, Bills, and Inflation over 52 Years," The Journal of Portfolio Management, Vol. 4, No. 4, 1978, pp. 18-19. doi:10.3905/jpm.1978.408655

[2] W. Reichenstein, "When Stock is Less Risky than Treasury Bills," Financial Analysts Journal, Vol. 42, No. 6, 1986, pp. 71-75. doi:10.2469/faj.v42.n6.71

[3] M. L. Leibowitz and T. C. Langetieg, "Shortfall Risk and the Asset Allocation Decision: A Simulation Analysis of Stock and Bond Risk Profiles," The Journal of Portfolio Management, Vol. 16, No. 1, 1989, pp. 61-68. doi:10.3905/jpm.1989.409236 
[4] C. K. Butler and D. L. Domian, "Long-Run Returns on Stock and Bond Portfolios: Implications for Retirement Planning," Financial Services Review, Vol. 2, No. 1, 1993, pp. 41-49. doi:10.1016/1057-0810(92)90014-4

[5] H. Bjorn and M. Persson, "Time Diversification and Estimation Risk," Financial Analysts Journal, Vol. 56, No. 5, 2000, pp. 55-62. doi:10.2469/faj.v56.n5.2390

[6] N. Strong and N. Taylor, "Time Diversification: Empirical Tests," Journal of Business Finance \& Accounting, Vol. 28, No. 3-4, 2001, pp. 263-302. doi:10.1111/1468-5957.00374

[7] K. Hickman, H. Hunter, J. Byrd, J. Beck and W. Terpening, "Life Cycle Investing Holding Periods and Risk," The Journal of Portfolio Management, Vol. 27, No. 2, 2001, pp. 101-111. doi:10.3905/jpm.2001.319796

[8] C. Gollier, "Time Diversification, Liquidity Constraints, and Decreasing Aversion to Risk on Wealth," Journal of Monetary Economics, Vol. 49, No. 7, 2002, pp. 14391459.

[9] T. S. Howe and D. L. Mistic, "Taxes, Time Diversification, and Asset Choice at Retirement," Journal of Eco- nomics and Finance, Vol. 27, No. 3, 2003, pp. 404-421. doi:10.1007/BF02761574

[10] B. Guo and M. Darnell, "Time Diversification and Long-Term Asset Allocation," The Journal of Wealth Management, Vol. 8, No. 3, 2005, pp. 65-76. doi:10.3905/jwm.2005.598423

[11] S. Mukherji, "A Study of Time Diversification with Block Bootstraps and Downside Risk," The Business Review, Vol. 10, No. 1, 2008, pp. 55-60.

[12] L. Alles, "The Cost of Downside Protection and the Time Diversification Issue in South Asian Stock Markets," Applied Financial Economics, Vol. 18, No. 10, 2008, p. 835. doi:10.1080/09603100701222333

[13] K. Panyagometh, "The Earlier You Invest, the Lower Probability of Ruin You Will Get," Competitiveness Review by NIDA Business School, in Thai, Vol. 1, No. 4, 2009, pp. 75-85.

[14] K. Singh, "On the Asymptotic Accuracy of Efron's Bootstrap,” Annals of Statistics, Vol. 9, 1981, pp. 1187-1195. doi:10.1214/aos/1176345636 